\title{
Change in straight ahead during adaptation to prism'
}

Samuel C. MeLaughlin and Kenneth I. Rifkin

\section{Abstraet}

The course of adaptation to prism was examined by recording S's localization of (a) an object which was objectively straight ahead and (b) straight ahead. It was found that, as the object of regard changed in apparent position and came to be localized correctly, straight ahead changed concomitantly with it. Mean data for $20 \mathrm{Ss}$ with a 25 diopter prism showed that, of the total change in localization during adaptation to the prism, $71 \%$ resulted from a concomitant change in the test stimulus and straight ahead, while only $29 \%$ could be attributed to a reduction in the disparity between the test stimulus and straight ahead.

\section{Introduction}

If an individual has one eye occluded and a prism in front of his other eye, the object of regard will be optically displaced. This can be demonstrated by having him reach for or point toward the object under conditions such that he cannot see his hand. He will be inaccurate in reaching or pointing, the direction and magnitude of his error being determined (within a margin of error) by the orientation and deviation-angle of the prism.

Over one hundred years ago, Czermak (1863) showed that the error in visual localization produced by a prism could be eliminated by having $S$ touch the object of regard repeatedly while seeing both his hand and the object. In the present experiment, this phenomenonprism adaptation-was investigated by following the course of adaptation in terms of S's localization of (a) an object which is straight ahead, and (b) straight ahead.

\section{Apparatus}

The visual display consisted of a vertical luminous white line $15 \mathrm{~cm}$ high and $0.2 \mathrm{~cm}$ wide, seen against a uniform black background at a viewing distance of 33 $\mathrm{cm}$ and equidistant from S's two eyes. The bottom of the line was $6 \mathrm{~cm}$ below S's horizontal line of sight. The $\mathrm{S}$, using a chin rest, had his left eye occluded and a 25 diopter prism base in (i. e., base to the left) before his right eye. (A 25 diopter prism produces a deviation of $8.33 \mathrm{~cm}$ on a tangent scale $33 \mathrm{~cm}$ distant.)

The apparatus was in a darkened room. Two lamps, shielded from S's direct view, illuminated the visual display. These lamps could be turned on and off by the experimenter. With the lamps on, $\mathrm{S}$ could see his hand and the pointer as well as the line. With the lamps off, he could see only the line.

The S's task was to move a pointer along a horizontal track perpendicular to his line of sight. The pointer was tapered upward, and its tip was $0.1 \mathrm{~cm}$ below the

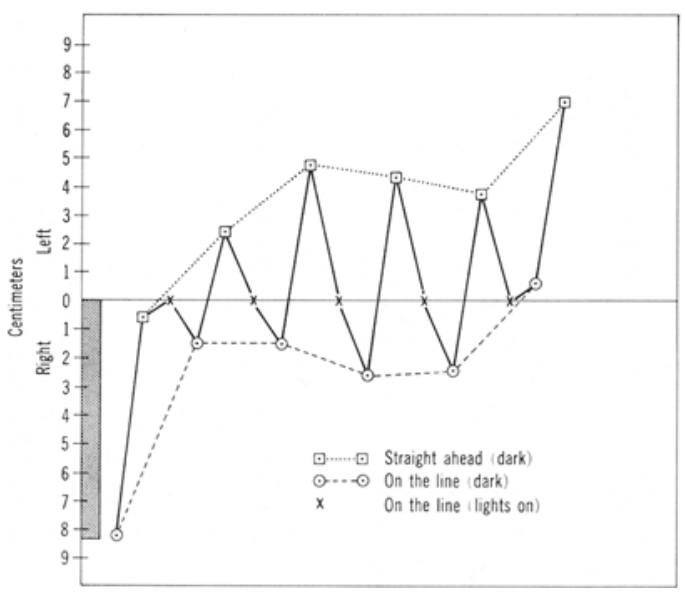

Fig. 1. Results for one S. Each point represents one setting of the pointer. The zero point on the ordinate represents the actual position of the stimulus line. Points above zero represent settings to S's left; points below zero represent settings to S's right. The thickened portion of the ordinate represents the direction and magnitude of the deviation produced by the prism. The solid line connects consecutive pointer settings. The accuracy of "line" settings with the light on is attributable to the fact the $\mathrm{S}$, being able to see both pointer and line, could align them precisely.

vertical white line. The experimenter was able to read the position of the pointer to the nearest $0.1 \mathrm{~cm}$ to right or left of the center of the line.

\section{Procedure}

Prior to each experiment, S's inter-pupillary distance was measured by a standard clinical technique (Kramer, 1953), and the horizontal distance between the prism and the chin rest was adjusted. The height of the chin rest was adjusted, and $\mathrm{S}$ was shown how to move the pointer.

During each experiment, $S$ made two types of settings: "on the line," in which the aim was to place the pointer directly beneath the line; and "straight ahead," in which the aim was to place the pointer exactly straight ahead. Two settings ("line" and "straight ahead") in the dark were followed by one setting ("line") with the lights on. (As in Czermak's original observation, the settings in which $\mathrm{S}$ was unable to see his hand constituted measurements of localization; and the settings with hand visible were adaptation trials.) This sequence was repeated, without interruption, until S's 'line"' setting in the dark was within $0.5 \mathrm{~cm}$ of the center of the line-i. e., until the error in localization had been reduced to $6 \%$ of the prism deviation. If $\mathrm{S}$ had not achieved this criterion after 20 adaptation trials, the experiment was terminated. 


\section{Results}

Figure 1 shows the results of the experiment for one S. The results for 20 Ss were as follows:

(1) The mean number of adaptation trials was 7.1 (range 2-14). Two Ss, whose data are not included in the mean values presented here, failed to achieve the criterion of adaptation after 20 adaptation trials.

(2) The mean value of the first "line" setting (prior to any adaptation trials) was $7.68 \mathrm{~cm}(\mathrm{SD}=2.07 \mathrm{~cm})$ to S's right.

(3) The mean vlaue of the first "straight ahead" setting was $0.62 \mathrm{~cm} \quad(\mathrm{SD}=2.24 \mathrm{~cm})$ to $\mathrm{S}$ 's right.

(4) The mean change in "line" settings during the course of the experiment was $7.54 \mathrm{~cm}(\mathrm{SD}=2.21 \mathrm{~cm})$ toward S's left.

(5) The mean change in "straight ahead" setting during the course of the experiment was $5.37 \mathrm{~cm}$ $(\mathrm{SD}=1.56)$ toward S's left.

\section{Diseussion}

Adaptation to prism is represented by the change in "line" settings during the course of the experiment (for $20 \mathrm{Ss}, \mathrm{M}=7.52 \mathrm{~cm}$ ). Of this total change, $5.36 \mathrm{~cm}$, or $71 \%$, resulted from a concomitant change in "line" and "straight ahead"' settings, while the remaining 2.16 $\mathrm{cm}(29 \%)$ resulted from a reduction in the disparity between the two kinds of settings. In other words, adaptation to prism (under the conditions of this experiment) is carried out chiefly by means of a concomitant change in the test stimulus and straight ahead, and only secondarily by a change in the position of the test stimulus with respect to straight ahead.

The consequence of the change in straight ahead is that $\mathrm{S}$, even though localizing a straight-ahead object correctly (i. e., being able to indicate its position accurately without seeing his hand or the pointer), does not perceive it as being straight ahead. Obviously, this is an incomplete adaptation to prism. An observer with only this much adaptation would have difficulty in walking across a room while wearing the prism. As soon as he tried to walk straight ahead, or to approach some object which appeared to him to be straight ahead, he would walk in some direction that differed from his objective straight ahead.

Evidently, then, complete adaptation to prism consists of at least two stages: (a) a concomitant change in straight ahead and the visual display, and (b) elimination of the disparity between the two.

These results also show that adaptation to prism is far from being a mere reversal of the effect of prism. The effect of prism (prior to any adaptation) is to change the apparent position of the visual display, leaving the straight ahead little if any affected (this is shown by the first two settings). By contrast, the first stage in the adaptive process is a concomitant change in straight ahead and the visual display.

To account for these findings, the first thing to be noted is that straight ahead is a spatial direction of reference which is common to all sensory modalities and to voluntary action as well. It is reasonable, therefore, that straight ahead (not being a visual phenomenon) should be little affected by prism, for the effect of prism is confined to the visual modality. The adaptive process, however, is not. Adaptation to prism is a process in which a new pattern of voluntary behavior becomes associated with a specific visual impression. This adjustment necessarily involves the perceptual construct which links vision and voluntary action: straight ahead must be affected.

Another characteristic of straight ahead is that it is a part of the spatial frame of reference within which $S$ localizes objects. Therefore, a change in straight ahead without a concomitant change in the position of a perceived object must be interpreted as dual event: (a) a change in the frame of reference; and (b) a change in the position of the object within the frame of reference. On the other hand, a concomitant shift in straight ahead and the object is a simpler phenomenon-namely, a change in the frame of reference only.

The present results show, then, that the first stage in adaptation to prism is a shift in egocentric frame of reference, the visual display retaining, for the most part, the displacement within that frame of reference which was imposed on it by the prism. The most significant fact about perceptual adaptation which these results convey is that it is somehow easier or more economical for the organism to shift its entire egocentric frame of reference than to change the position of the visual display with respect to straight ahead.

\section{References}

CZERMAK, J. Wiener Berichte XVII (1863). Pp. 575-577. Cited at length in Helmholtz, Physiological optics. (3rd ed.), translated by J. P. C. Southall. The Optical Society of America, George Banta Publishing Co., Menasha, Wisconsin, 1925. Vol III. P. 246. KRAMER, M. E. Clinical orthoptics. (2nd ed.) St. Louis: The C. V. Mosby Co, 1953, P. 223.

Note

1. The research described in this paper was supported by PHS Research Grant NB-04540-03 from the Division of Neurological Diseases and Blindness, Public Health Service. 